\title{
Executive functions in the context of complex learning: Malleable moderators?
}

\author{
Matthias Schwaighofer ${ }^{1}$, Markus Bühner, Frank Fischer \\ University of Munich, Germany \\ Article received 19 August / revised 4 January / accepted 9 January / available online 15 February
}

\begin{abstract}
Executive functions are crucial for complex learning in addition to prior knowledge. In this article, we argue that executive functions can moderate the effectiveness of instructional approaches that vary with respect to the demand on these functions. In addition, we suggest that engagement in complex activity contexts rather than specific cognitive training paradigms may enhance executive functions and yield practically relevant transfer effects to other cognitive abilities. We develop several hypotheses and principles for how to improve executive functions in these contexts. For future research, we suggest to systematically investigate the moderating role of executive functions in learning environments with varying degrees of instructional support and varying context characteristics. We identify potential factors influencing the improvement of executive functions to be considered in a systematic research program.
\end{abstract}

Keywords: Executive functions; complex learning; moderation; training

${ }^{1}$ Corresponding author: Matthias Schwaighofer, Department of Psychology, Ludwig-Maximilians-Universität München, Leopoldstraße 13, 80802 Munich, Germany. Email: M.Schwaighofer@psy.lmu.de DOI: http://dx.doi.org/10.14786/flr.v5i1.268 


\section{Problem}

In recent research on learning and instruction, learners' prior knowledge and working memory capacity are considered crucial prerequisites for complex learning. It is widely accepted that prior knowledge is variable. Working memory is usually considered to be limited and constant apart from developmental and intra-individual variation (e.g., due to task demands; Trezise \& Reeve, 2014). Moreover, the interplay of prior knowledge and working memory is typically considered to be the main determinant of learning in complex learning environments.

Two recent patterns of findings in other areas of research may challenge this apparent consensus. First, working memory is but one from a set of basic cognitive functions called executive functions (Andersson, 2010; Miyake \& Friedman, 2012). There is evidence that other executive functions are highly relevant for complex learning as well. Second, the view that working memory and other executive functions cannot be altered substantially, except through developmental changes and apart from intra-individual variations, has been challenged by recent studies.

In this article we link these two bodies of research and delineate the impact of an innovative framework for research on learning and instruction.

\section{Executive functions in the context of complex learning}

\section{1 Definition of executive functions}

Executive functions are basic cognitive functions (Andersson, 2010) and as such are typically conceived as effective across domains (Friedman \& Miyake, 2017). Executive functions receive attention due to their strong relation to self-control (also referred to as self-regulation) (Miyake \& Friedman, 2012). Self-control is considered important for numerous outcomes relevant to daily life, including learning (e.g., Blair, Ursache, Greenberg, Vernon-Feagans, \& The Family Life Project Investigators, 2015; Mischel et al., 2011). The literature on executive function typically focusses on working memory, shifting, and inhibition as core executive functions (for an overview see Miyake \& Friedman, 2016).

Working memory is used to store and manipulate information temporarily (Baddeley, Allen, \& Hitch, 2011). Working memory processes typically involve retrieval and transformation of information from long-term memory and from the environment. These processes are strongly dependent on the capacity of working memory (Ecker, Lewandowsky, \& Oberauer, 2014; Ecker, Lewandowsky, Oberauer, \& Chee, 2010). The capacity of working memory is limited to about four chunks (collections of concepts with strong associations), on average (Cowan, 2001). Individual capacity limits can differ widely, ranging from 2 to 6 chunks (Cowan, 2001). Individual differences in working memory capacity may be explained by three mechanisms, which include maintenance of information in short-term storage, retrieving information from long-term memory, and attentional control (Shipstead, Lindsey, Marshall, \& Engle, 2014). Working memory capacity seems to be crucial for complex learning (e.g., Sweller, 2011). However, capacity is not the only aspect of working memory that may be relevant for complex learning. Updating is sometimes also considered to be an important aspect of working memory. Updating involves the continuous monitoring and adding or removal of content in working memory (Miyake, Friedman, Emerson, Witzki, \& Howerter, 2000). Based on three experiments, Ecker et al. (2014) conclude that the removal of irrelevant information from working memory is the crucial part of substitution, hence also for working memory updating. Retrieval and transformation of information from long-term memory as two further updating processes have found to be strongly related to working memory capacity (Ecker et al., $2014 ; 2010)$. Converging evidence indicates that updating and working memory capacity are both strongly related to each other (e.g., Wilhelm, Hildebrandt, \& Oberauer, 2013; Schmiedek, Hildebrandt, Lövdén, Wilhelm, 
\& Lindenberger, 2009; Schmiedek, Lövdén, \& Lindenberger, 2014). In a nutshell, updating processes seem to be largely related to working memory capacity that is considered to be crucial for complex learning (see section 2.2.). Therefore, for research on complex learning we suggest putting an emphasis on working memory capacity. This emphasis does not however, imply that updating-specific processes, like the removal of items from working memory, are irrelevant for complex learning.

Shifting, also denoted as attention switching, refers to the ability to switch flexibly between multiple representations and strategies with changing task demands (Miyake et al., 2000). Inhibition is the ability to overcome dominant or prepotent responses (Miyake \& Friedman, 2012), which enables an individual to avoid acting on the first reaction that comes to mind. Inhibition may be involved in working memory and shifting processes. For example, the intent to store only information of a given text in working memory presumably requires inhibition of interference from other sources of information (e.g., thoughts unrelated to the text). Switching attention between different parts of a text requires inhibition of previous parts of the text. For relatively simple tasks, studies have shown that there is no specific variance left for inhibition controlling for a common factor extracted from working memory and shifting (e.g., Miyake \& Friedman, 2012). In addition, for several approaches to measuring inhibition the construct was deemed to be multi-dimensional (e.g., Friedman \& Miyake, 2004; Krumm et al., 2009). Despite these findings, inhibition seems to be important for many processes requiring working memory and shifting and may explain variance in the context of complex learning. Thus, we consider the role of the executive function of inhibition for complex learning in our framework. In the remainder of the article, the term executive functions refers to working memory, shifting and inhibition whereas the term cognitive functions also includes cognitive abilities or skills such as fluid intelligence and long-term memory.

\subsection{Relevance of executive functions for complex learning}

A main instructional goal with complex learning is to simultaneously facilitate conceptual learning in a domain and the development of skills. Complex learning typically provides the learner with non-trivial activities related to inquiry, design or problem solving in real domains. Learners' appropriate engagements in these activities require the coordinated application of domain concepts as well as strategies or skills (see also van Merriënboer \& Kirschner, 2012, p. 2). In the following, we argue that the executive functions of working memory, shifting, and inhibition are important for complex learning.

In general, the executive function of working memory seems to be crucial for learning because it provides a mental workspace in which information can be held while carrying out other activities (Gathercole \& Alloway, 2007). Working memory capacity is considered to be important for complex learning within cognitive load theory (e.g., Sweller, 2011). According to cognitive load theory, the role of working memory capacity is connected to the element interactivity of learning material. Elements are information to be learned or information that has been learned (e.g., a concept; Sweller, 2010). Collections of interacting elements can therefore be conceived as chunks. Learning material with many interacting elements, which cannot be learned in isolation, is high in element interactivity and induces a high load on one's central executive function, namely working memory. Interacting elements that have to be simultaneously processed in working memory may be unrelated to learning relevant information. However, the interacting elements in relevant learning material can be crucial to fostering understanding (Sweller, 2010). Thus, we consider complex learning material as being high in element interactivity.

Working memory capacity is assumed to play an important role in dealing with element interactivity in fostering learning. Connecting elements to units (i.e., chunks) can reduce the number of elements required for complex learning that have to be held in working memory. Whether elements can be grouped to units depends on the amount of prior knowledge. Learners with high prior knowledge in a domain hold schemas in long-term memory which can be represented as single elements in working memory and therefore induce low working memory load (Sweller, 2011). In contrast, for learners with low levels of prior knowledge, complex problem solving can induce a high load in working memory (Schnotz \& Kürschner, 2007). Thus, not enough working memory capacity can be devoted to making sense of meaningful material (Sweller, 2011) and small learning 
gains may result (e.g., Sweller, Mawer, \& Howe, 1982). Therefore, in the framework of cognitive load theory, instructional methods can be improved with respect to their effectiveness and efficiency if they take into account the individual limits of working memory capacity (Sweller, 2011). Individual differences in working memory capacity have been linked to the variability in several complex learning outcomes. For example, working memory capacity has been shown to be associated with math achievement (Peng, Namkung, Barnes, \& Sun, 2016), reading comprehension (Daneman \& Merikle, 1996), accomplishment in chemistry (e.g., Tsaparlis, 2005), and problem solving (e.g., Bühner, Kröner, \& Ziegler, 2008). Beyond these associations with complex learning outcomes, working memory capacity is moderately correlated with fluid intelligence (e.g., Ackermann, Beier, \& Boyle, 2002; Redick, Unsworth, Kelly, \& Engle, 2012).

Compared with research on working memory, the interest in the relevance of shifting, as an executive function for cognitive achievements, has grown only over the last years. Research suggests that shifting is relevant for tasks that require switching between different aspects of a problem (e.g., Agostino, Johnson, \& Pascual-Leone, 2010; Blair, Knipe, \& Gamson, 2008). For example, solving mathematical problems involving multiple equations may require switching attention between equations or parts of them.

Meta-analyses indicate that shifting is associated with reading and math achievements as complex learning outcomes (Yeniad, Malda, Mesman, van Ijzendoorn, \& Pieper, 2013). However, the meta-analysis by Yeniad et al. (2013) did not control for other cognitive functions that could explain the associations between shifting and mathematics achievement (Bull \& Lee, 2014). In addition, the studies included in the meta-analysis by Yeniad et al. (2013) were conducted on children's executive functions, which is of note because there is evidence that measures of executive functions do not clearly separate all executive functions prior to the age of 15 years (Lee, Bull, \& Ho, 2013). The role of shifting for academic achievements in adulthood is not yet clear. Some indications could be taken from research showing a relationship of common executive functioning assessed by one measure in early childhood and a shifting factor in adolescence (Friedman, Miyake, Robinson, \& Hewitt, 2011). In addition, some studies demonstrate that working memory can already be separated from shifting in late childhood and executive functions show considerable stability from adolescence into early adulthood (Friedman et al., 2016). Given the stability of shifting and its putative relevance for academic achievements in childhood, we submit that shifting may also be relevant for complex learning of older children, adolescents and adults.

With respect to the executive function of inhibition, several primary studies show the association of inhibition and mathematics achievement (e.g., Espy, McDiarmid, Cwik, Stalets, Hamby, \& Senn, 2004; Lan, Legare, Ponitz, Li, \& Morrison, 2011). Results of a meta-analysis by Jacob and Parkinson (2015) indicate that inhibition is related to math and reading achievements in addition to shifting and working memory. Notably, at least for mathematics achievement, it remains unclear whether the role of inhibition depends on updating. Several studies found only updating to be associated with math achievement and inhibition was only related to math achievement when there was no control for updating (for an overview, see Bull \& Lee, 2014). However, there are also arguments that consider inhibition as the common factor of executive functions (Miyake \& Friedman, 2012), which could explain associations between working memory capacity and shifting on the one hand and cognitive achievements on the other hand.

In sum, individual differences in working memory, shifting, and inhibition are important for explaining variance in various cognitive achievements related to complex learning. As will be discussed in the next section, interactions among these individual differences and different instructional approaches for complex learning have hardly been investigated systematically.

\subsection{Aptitude treatment interactions in the context of complex learning}

Research on aptitude treatment interactions (ATIs) (e.g., Snow \& Lohman, 1984) suggests that the effectiveness of instructional approaches depends on learning prerequisites. The prerequisite that is typically investigated in the context of complex learning is prior knowledge (e.g., Kester \& Kirschner, 2012). This is not surprising given that the acquisition of domain-specific schemas can be considered a main goal in complex 
learning environments (Kalyuga \& Singh, 2015) and prior knowledge is regarded as a strong predictor of learning (e.g., Dochy, Segers, \& Buehl, 1999). The moderating influence of prior knowledge on the effectiveness of various instructional supports has been demonstrated frequently. A general finding of this research is that the benefits of instructional support decrease with increasing prior knowledge. This pattern of results has been termed the expertise reversal effect (Kalyuga, 2007) and has been found for several instructional approaches, including instructional support using animations, simulations, and hypermedia-based materials (e.g., Kalyuga, 2013; Kalyuga, Rikers, \& Paas, 2012).

Executive functions have seldom been measured as potential moderators in research on ATIs despite their potential relevance for complex learning. Perhaps most surprisingly, the executive function of working memory capacity has rarely been assessed as a potential moderator using objective, reliable, and valid measures. De Jong (2010) lists some studies as exceptions, but in these as well as more recent studies (e.g., Lehmann, Goussios, \& Seufert, 2016; Park, Korbach, \& Brünken, 2015), working memory was measured by using only one single task. Hence, task-specific influences are not eliminated (Schwaighofer, Bühner, \& Fischer, 2016). Furthermore, in some of these studies, working memory capacity has been included as a control variable and not as a moderator variable (e.g., Berends \& van Lieshout, 2009; Park et al., 2015). Working memory capacity might moderate the effects of different kinds of instructional support aimed at reducing working memory load. Instructional support through worked examples is assumed to be effective in reducing load in working memory (e.g., Renkl, 2014). The benefit of worked examples might be especially pronounced for learners with low working memory capacity (van Gog \& Rummel, 2010).

Yet, shifting might also moderate the effectiveness of different kinds of instructional support. The moderating role of shifting may depend on context characteristics such as task complexity and their demand for shifting. Unsupported problem solving, which requires switching between different sources of information (e.g. within a problem or between problem and additional information), may be overwhelming for learners with low shifting ability. These learners may benefit from instructional support that reduces the demands on shifting (e.g. by integrating information from different sources). In contrast, learners with high shifting ability may not benefit from such instructional support because they can deal with high shifting demands. In a recent study, Schwaighofer et al. (2016) investigated the moderating role of the executive functions of working memory capacity and shifting as well as fluid intelligence for the effectiveness of worked examples versus problem solving. Compared to problem solving, worked examples had lower benefits the higher the shifting was. Presumably, problem-solving puts a high demand on shifting ability.

Inhibition may moderate the effectiveness of various instructional approaches, especially when these approaches differ in the need for inhibiting responses or resisting interference (e.g., from material irrelevant to learning). Furthermore, inhibition may influence the potential moderating role of working memory and shifting due to the involvement of inhibition in working memory and shifting processes. For example, shifting among different information relevant to solving a problem may involve inhibiting irrelevant information. The inhibition of information unrelated to problem-solving may also free up working memory capacity to deal with relevant information (see also Trezise \& Reeve, 2014). Learners with high inhibition ability may have few difficulties dealing with problems with high demands on this executive function. Therefore, these learners may benefit less from instructional support that lowers these demands.

Besides these executive functions, other cognitive functions may moderate the effectiveness of different kinds of instructional support. One further potential moderator is fluid intelligence, defined as the "ability to reason and to solve new problems" (König, Bühner, \& Mürling, 2005, p. 245). Fluid intelligence is not as fundamental when compared to executive functions and may be influenced by these functions (Diamond, 2013). The conceptualization of intelligence is based on implicit theories of researchers and their assumptions about different methods to test it (Oberauer, Schulze, Wilhelm, \& Süß, 2005). Nonetheless, fluid intelligence is worth considering in the context of complex learning along with executive functions for several reasons. Reasoning which information seems to be necessary to solve a novel, complex problem may be a cognitive process unique to fluid intelligence. Specifically, there is empirical evidence that fluid intelligence is especially important for solving novel and complex tasks (e.g., Primi, Ferrão, \& Almeida, 2010). Learners with low fluid intelligence 
may have severe difficulties figuring out which information is necessary to solve a novel problem. These learners may benefit from instructional support that reduces the demands on fluid intelligence (e.g. by providing information relevant to solve a problem at hand). Learners with high fluid intelligence do not need this instructional support because they can more easily use their reasoning capacity to determine which information is necessary to solve a given problem. In line with this argumentation, Schwaighofer et al. (2016) found that the benefits of worked examples compared to problem solving were lower the higher fluid intelligence was. In that study, the moderating role of fluid intelligence was independent of working memory capacity and shifting.

In summary, both executive functions as well as fluid intelligence are likely to moderate the effects of instruction on complex learning outcomes. These moderation effects could occur independent of prior knowledge or in addition to the moderating role of prior knowledge. However, these factors have hardly been studied systematically and in their interplay in research on complex learning.

Moreover, there are methodological concerns with respect to ATIs in the context of complex learning. In several analyses of ATIs, only global moderation effects of treatments on outcomes have been tested. Sometimes only high or low values in an aptitude such as prior knowledge or working memory capacity were compared (e.g., Kalyuga \& Sweller, Exp. 3, 2004; Seufert, Schütze, \& Brünken, 2009). The main problem of these comparisons and of testing only global moderation effects is that it is not known for which regions of values of a moderator the independent variable has a significant effect on the dependent variable. For example, the regions of values in prior knowledge for which the presence of worked examples has a significant effect on knowledge at post-test is unknown when only high or low values in prior knowledge are compared.

The moderating role of executive functions may additionally depend on context characteristics such as task complexity or time constraints. Complex tasks can vary in their demand on cognitive functions, and certain instructional support may reduce these demands. For example, shifting demands in a problem-solving condition may be reduced in the presence of worked examples (Schwaighofer et al., 2016). In addition to task complexity, other context characteristics such as time constraints may play a role for ATIs. In the study by Schwaighofer et al. (2016) the effectiveness of worked examples was not moderated by working memory capacity. One plausible reason could be that learners did not have to work under time pressure. Working memory overload may arise only under time pressure and when offloading (e.g., by using notepads) is prevented (de Jong, 2010). Thus, the moderating role of executive functions for the effects of instructional approaches on learning outcomes may depend on the context, such as whether learners have to work on tasks under time pressure.

In summary, we can say that executive functions have been shown to play an important role for complex learning. Specific moderation processes that may involve the interplay of executive functions have not yet been studied systematically in the context of instruction. In addition, instructional research, including measures of executive functions, has so far not fully used the methodological possibilities to objectively, reliably, and validly measure the constructs and explored their possible local moderation effects.

Implicitly, the instructional research reported in the preceding sections assumes stability of executive functions. However, in a different area of research, several intervention studies have shown that executive functions seem to be malleable to a certain extent (Melby-Lervåg, Redick, \& Hulme, 2016). We submit that this research has relevance for instructional research as well. In the next section, we outline research on the malleability of executive functions, focusing on approaches that may improve these functions.

\subsection{Malleability of executive functions}

\subsubsection{Developmental and further influences on executive functions}

Current theorizing and findings indicate that executive functions develop early in childhood, improve until adolescence, and then decline with age. In addition to age, factors such as stress, sadness, and loneliness can have deleterious effects on executive functions (for an overview, see Diamond, 2013). 
Importantly, some age-independent factors can lead to variation in executive functioning within small and longer periods of time. For instance, experiencing frustration in the classroom can lead to impaired executive functioning, especially the inhibition of prepotent responses, thus generating high intra-individual variation in this functioning (Pnevmatikos \& Trikkaliotis, 2013). Also, higher intrinsic motivation is associated with higher executive functions (Sosic-Vasic, Keis, Lau, Spitzer, \& Streb, 2015). Negative emotions such as anxiety may impair executive functions (Eysenck et al., 2007) but the extent of impairment may also depend on initial levels of executive functioning (working memory) and vary within short periods of time (Trezise \& Reeve, 2014). Perceived strain may also impair executive functions temporarily (e.g., Liston, McEwen, \& Casey, 2009); this holds for different age groups (Tun, Miller-Martinez, Lachman, \& Seeman, 2013).

\subsubsection{Approaches to improve executive functions}

Subsequently, we review existing evidence on the malleability of executive functions in what has been called narrow activity contexts (Schwaighofer, Fischer, \& Bühner, 2015) such as computerized training of working memory. These contexts involve material low in complexity such as colored squares. We then turn to conjectures concerned with the improvement of executive functions in more complex activity contexts.

Recently, a wealth of studies has investigated the malleability of executive functions by applying different training methods. The most popular approach involves computerized training of cognitive functions, particularly working memory training. The aim of working memory training and other approaches to train executive functions is to improve the targeted cognitive function and hence yield transfer effects to other cognitive abilities or skills. Although some primary studies yielded promising results (e.g., Jaeggi, Buschkuehl, Jonides, \& Perrig, 2008), recent meta-analyses cast doubts on the transfer of these effects to other cognitive abilities such as mathematical abilities (Melby-Lervåg et al., 2016; Schwaighofer et al., 2015). In support of these doubts, recent meta-analytic evidence (Karbach \& Verhaeghen, 2014; Melby-Lervåg \& Hulme, 2016) suggests that training of executive functions yields no substantial transfer effects to cognitive abilities that were not the focus of the training. This seems to hold, at least when training groups are compared with active control groups (e.g. groups completing physical exercises; Karbach \& Verhaeghen, 2014; Melby-Lervåg \& Hulme, 2016).

One reason for the lack of practically relevant transfer effects for computerized training of executive functions may lie in the nature of the narrow activity contexts. Within these narrow contexts, tasks only tap one or more executive functions in isolation. However, complex-learning tasks may require the well-coordinated interplay of several cognitive functions. For example, mathematical problem solving may tax the executive functions of working memory, inhibition, and shifting and may require their coordination. Solving a complex mathematical equation may involve keeping in mind relevant elements in the equation and additional information from texts. This constellation taxes working memory. Switching among various information sources to solve the equation might put a high demand on the shifting function. Resisting irrelevant information or distracting stimuli (e.g., a smartphone buzzing) taxes inhibition. Thus, working memory, shifting, and inhibition may have to operate in concert when solving the mathematical equations. Executive functions usually have to be used together and their interplay presumably has to be coordinated. For example, keeping in mind information from additional text while switching among information of the equation and resisting interference from a buzzing smartphone involves working memory capacity, shifting, inhibition, and presumably the coordinated interplay of these functions. The involvement of executive functions and their interplay in complex learning activities may depend on task demands. For example, Borella and Ribaupierre (2014) found that working memory predicts text comprehension when participants were allowed to look at the pertaining text. When participants were not allowed to look at the pertaining text, inhibition (resistance to distractor interference) predicts text comprehension in addition to working memory. The authors argue that preventing irrelevant information from entering working memory is particularly important when learners have to cope with information in addition to a text comprehension task (Borella \& Ribaupierre, 2014). Executive functions appear to be substantially correlated but also have a unique variance (Friedman \& Miyake, 2017). This unique variance of the different executive functions may explain why working memory and shifting can be simultaneously and uniquely related to complex academic achievements such as reading abilities (e.g., Jacobson et al., 2016). Activities such as solving 
mathematical problems or reading texts are often embedded in more complex contexts such as classroom instruction at schools. Complex activity contexts can be designed such that activities within these contexts improve executive functions and yield transfer effects to academic achievements (see Diamond \& Ling, 2016).

The demands on executive functions may depend on the problems or tasks used in complex activity contexts. One could hypothesize that the demands may be reduced or enhanced by varying the degree of instructional support. Evidence in support of this hypothesis would be the moderation effects executive functions have in learning environments with varying degrees of instructional support and demand on these functions. The moderating role would suggest that varying degrees of instructional support put different demands on executive functions. Therefore, a possible enhancement of executive functions can depend on the degree of instructional support. This has been labeled the "detrimental instruction hypothesis" (Schwaighofer et al., 2015), which states that instructional support that reduces the demands on executive functions can be detrimental with respect to improvements of these functions and transfer effects. This hypothesis is related to the assumption that executive functions have to be continuously challenged on a high level (i.e., tasks have to adapt to consistently guarantee a high demand on executive functions; Schwaighofer et al., 2015). Complementary to this assumption, in their comprehensive review of the literature on training of executive functions, Diamond and Ling (2016) note that to avoid frustrating or boring the learner, the challenge should not be too high or too low. In addition, the longer executive functions are challenged, the higher the likelihood of training effects. Training of executive functions probably should not pause too long because improvements in executive functions usually decline with increasing time lags between training sessions (Diamond \& Ling, 2016).

A further speculation concerning the improvement of executive functions in complex activity contexts has been labeled the "cognitive flexibility hypothesis". According to this hypothesis, executive functions have to be challenged in different contexts to yield transfer of improved executive functioning to other tasks (Schwaighofer et al., 2015). The cognitive flexibility hypothesis is based on research suggesting that learning in multiple contexts can foster transfer (e.g., Spiro, Coulson, Feltovich, \& Anderson, 1988). In multiple complex activity contexts, executive functions might be trained with respect to their coordinated interplay in order to adapt to different complex tasks. Thus, executive functions and their interplay may be trained to adapt to different demands. As a result, executive functions may not only improve, but also the chance to yield transfer effects to other complex tasks requiring executive functions and their interplay might increase. In contrast to isolated computerized trainings of executive functions, complex tasks provide the additional opportunity to acquire relevant knowledge of a certain domain.

Moreover, two principles for the training of executive functions postulated by Diamond and Ling (2016) deserve attention. First, these authors argue that people with the poorest executive functions seem to gain most from training programs. This principle is based on findings from various intervention studies, ranging from working memory training (e.g., Holmes et al., 2010) to physical training (Kramer \& Erickson, 2007). Of particular interest is the study by Blair and Raver (2014), in which a comprehensive curriculum (Tools of the Mind), shown to be effective in an earlier study (Diamond, Barnett, Thomas, \& Munro, 2007), was implemented in kindergarten classes for two years. In this curriculum, activities designed to improve executive functions are intertwined with curricular learning activities. Teachers are trained to organize and manage instruction in order to improve children's self-regulation skills through focused interactions with their classmates. Because executive functions seem to be important for self-regulation (e.g., Blair \& Ursache, 2011), activities intended to foster selfregulation skills may improve executive functions.

The Tools of the Mind curriculum also includes individualized, differentiated scaffolding depending on the mastery of specific skills. In addition, the children meet with teachers on a weekly basis to reflect on their learning and to develop metacognitive strategies. In contrast to the study by Diamond et al. (2007), the study by Blair and Raver (2014) included baseline measures of executive functions to rule out effects due to pre-existing differences in executive functions. Children in the treatment group showed improvements in executive functions and outcomes such as reading and math. The effect sizes for several outcomes, such as measures of executive functions and reasoning were highest for low-income children assumed to be disadvantaged with respect to the development of their executive functions (Blair \& Raver, 2014). According to the second principle postulated by 
Diamond and Ling (2016), effects of training are especially pronounced for measures that push the limits of participants' executive functions. In a study by Diamond et al. (2007), the benefits of the Tools of the Mind curriculum were largest for the executive function tasks with the highest demands. A possible extension of the principle proposed by Diamond and Ling (2016) refers to the nature of tasks used to assess transfer effects following improvements in executive functions. It is possible that transfer effects are also larger for transfer tasks that put high demands on executive functions. Support for this assumption comes from research indicating that executive functions better predict higher than lower level academic skills (e.g., Jacobson et al., 2016). Tasks used to measure higher academic skills may better capture individual differences in executive functions than transfer effects on lower academic skills. Therefore, these tasks could also be more appropriate for measuring transfer effects following improvements of executive functions.

The principles and hypotheses mentioned above can be seen as complementary. We assume that the more facilitative principles are considered, the more effective the training of executive functions will be. Also, the opportunity to transfer effects to other cognitive abilities (e.g., the ability to solve complex academic tasks in a domain) will increase the more principles are considered.

\section{Future directions for research on complex learning and executive functions}

\subsection{Investigating the moderating role of executive functions in learning environments with varying}

\section{demands on these functions}

In studies on complex learning, the moderating role of learning prerequisites has mainly been analyzed for prior knowledge. Different levels of prior knowledge have been linked to varying demands on working memory. Working memory demands are lower for persons with high prior knowledge due to a reduced number of elements which have to be stored in working memory or a reduced level of element interactivity, respectively (Sweller, 2011). As a consequence, working memory may not moderate the effectiveness of varying degrees of instructional support when learners already dispose of high prior knowledge. Within the context of complex learning, interactions between prior knowledge and working memory have been considered to be crucial aspects of the human cognitive architecture. However, research on executive functions indicates that shifting and inhibition can also be relevant for complex learning and potentially moderate the effectiveness of instructional approaches. In addition to working memory, the moderating influence of shifting and inhibition may also depend on prior knowledge. High prior knowledge learners may be better able to quickly identify relevant information to solve a problem. Thus, these learners do not have to frequently shift among potentially relevant and irrelevant information to solve a problem. Furthermore, learners with high prior knowledge do not have to inhibit a large amount of irrelevant information. Nonetheless, for learners with high prior knowledge the demands on executive functions could be high under certain conditions, which have to be systematically explored. Executive functions may moderate the effectiveness of instructional approaches varying in the demands on executive functions. Accordingly, instructional approaches could be effectively adapted to consider different levels of executive functions. For example, presenting information relevant for solving a problem together with the problem may lower the requirement to integrate spatially separated information. Therefore, the demand on working memory may be reduced. Executive functions have been included in only a few studies and have not been measured on a latent variable level. Reliable and valid measures of executive functions, however, have recently been established (e.g., Friedman et al., 2016; Redick, Broadway et al., 2012).

Thus, we propose that executive functions receive more attention in the context of complex learning. Moreover, fluid intelligence is worthwhile to consider in addition to executive functions because it is presumably important for solving novel complex problems. Notably, we do not propose considering the moderating role of executive functions or other cognitive abilities such as fluid intelligence instead of prior knowledge. Rather, we 
suggest systematically addressing the conditions for interactions between prior knowledge and executive functions with respect to their moderating role for the effects of varying instructional approaches. More specifically, we suggest that future research should consider the following three recommendations.

a) Exploring the conditions for moderation effects in the context of complex learning.

To systematically address the potential moderating role of prior knowledge and executive functions, we propose exploring the conditions for moderation effects of one or several cognitive prerequisites. Examples of these conditions or context characteristics may include the complexity of tasks or time constraints. For instance, in a hypothetical scenario learners may have to frequently switch their attention between information from different sources (e.g., different websites or texts) and task instruction to solve a challenging problem. In order to better focus on one source of information after a shift of attention, learners have to inhibit irrelevant information from previous sources (e.g., interesting details of a text that are not relevant for a problem at hand). Under these conditions or context characteristics, learners with low shifting ability and/or low inhibition ability in particular could be overstrained. Instructional approaches as worked examples may lower the demands on shifting ability and inhibition. Providing solution steps (parts of worked examples) for complex tasks may reduce the necessity to switch attention between information from different sources and therefore, reduce shifting demands. Moreover, fewer necessary shifts among relevant and irrelevant information can also reduce the demand for inhibiting irrelevant information. Learners with low shifting and inhibition ability would benefit from this instructional support. Thus, we hypothesize that the executive functions of shifting and inhibition may moderate the effects of the instructional approach (i.e., presence of worked examples) on learning outcomes.

It is important to note that the moderating role of executive functions presumably depends on the differences between instructional approaches in their demands on these functions. Shifting demands may be high in conditions with low and high degrees of instructional support if both conditions require frequent switching between several information sources to meet task demands. The high degree of instructional support may not significantly reduce the demand on shifting, and learners with low shifting ability will presumably not benefit from the instructional support. Thus, shifting ability may not moderate the effect of the degree of instructional support on learning outcomes under these circumstances. Accordingly, we hypothesize that executive functions moderate the effects of instructional approaches on learning outcomes only if these approaches significantly differ in their demands on executive functions. It would be a worthwhile task to review different instructional approaches like learning-by-design, inquiry learning, problem-based learning, direct instruction, and worked examples with respect to their typical demands on the different executive functions and their interplay.

Knowledge on moderation effects can be helpful for selecting or designing particular instructional approaches. A moderating role of executive functions for the effectiveness of different instructional approaches suggests that these approaches differ with respect to the demands on executive functions. Approaches with high demand on executive functions may only be beneficial for learners with high executive functions. Instructional approaches might be adapted to meet learners' executive functions or other potential moderators, such as prior knowledge and fluid intelligence. One aim of adapting instructional approaches would be to foster domain knowledge by providing adequate support. A second aim is connected to the training of executive functions by establishing and maintaining an optimal level of demand on executive functions. We elaborate on this second aim in section 3.2 .

b) Measuring executive functions on a latent variable level.

In studies investigating interactions between working memory and instructional approaches, working memory has predominantly been measured by using single tasks. The executive function of shifting has rarely been measured in research on ATIs. We propose to measure executive functions on the latent variable level using several tasks for each cognitive function to minimize task-specific variance. Shifting as well as working memory capacity and inhibition can be measured with three computerized tasks for each construct (e.g., Friedman et al., 2016; Redick, Broadway, et al., 2012). In the case of working memory, even short versions allow assessment of working memory capacity on a latent variable level within about 25 minutes (Oswald, McAbee, Redick, \& Hambrick, 2015). In these working memory tasks, participants have to complete series of process-storage 
sequences (e.g., solve a simple mathematical equation and store a letter) and afterwards recall the stimuli (e.g., the letters) (Oswald et al., 2015). In typical shifting tasks, participants have to flexibly switch between two tasks, for instance by indicating the shape or the color of a stimulus. A cue presented before the stimulus indicates which task to perform. Shifting can be measured as the difference in mean reaction times between correct switch and no-switch trials. In no-switch trials, the same task has to be performed on consecutive tasks (i.e., the same cue appears consecutively). In switch trials, the task changes from one trial to the subsequent one (i.e., the cue changes) (Friedman et al., 2011). Notably, recent research proposes applying alternative scoring methods to measure shifting. One of these alternative methods involves calculating bin scores. Bin scores consider the speed of switching between switch and no-switch trials and accuracy. Traditional difference scores do not consider the accuracy when switching between two tasks (Draheim, Hicks, \& Engle, 2016). Thus, difference scores only reflect the speed of switching between two tasks but do not account for the number of errors or correct shifts respectively. A frequently used task to measure inhibition is the antisaccade task (e.g., Friedman et al., 2016). This task requires participants to correctly identify a target stimulus by inhibiting the tendency to look at a cue previously presented at a different location as the target stimulus. The dependent variable for the antisaccade task is typically the proportion of correct responses (Friedman et al., 2008). Every task for measuring a single executive function involves specific content material. Hence, there is systematic variance due to the task context. To alleviate the influence of the task context, several tasks should be used to measure executive functions on a latent variable level (see also Schwaighofer et al., 2016; Miyake \& Friedman, 2012).

c) Applying more appropriate statistical analyses.

Finally, moderator analyses in the context of complex learning may benefit from recent methodological advances. Analyses of moderated moderation effects are feasible with recent analysis techniques (Hayes, 2013). These tools also allow, for example, the application of the so-called Johnson-Neyman technique, which makes it possible to analyze the effects of an independent variable on a dependent variable for different ranges of values of one or two continuous moderators. Hence, local moderations can be identified with no loss of information compared to categorizing continuous moderators. Notably, categorical moderators and covariates can also be included in moderation analyses. For example, the shared variance of fluid intelligence and working memory can be controlled for. Thus, the unique contributions of single moderators can be identified in the complex pattern of effects for several cognitive functions.

The moderating role of executive functions could provide information about the conditions under which complex learning activities put lower or higher demands on executive functions. This information is connected to the idea of training executive functions in complex activity contexts over longer periods of time. Complex learning activities with high demands on executive functions and their coordinated interplay may be more likely to improve these functions. We propose considering the development of executive functions in complex activity contexts in addition to fostering knowledge acquisition. Executive functions may improve in complex activity contexts and therefore acquiring domain knowledge may not be the sole aim for complex learning.

\subsection{Promising routes for training of executive functions in complex activity contexts}

Cognitive abilities for solving complex academic tasks likely require not only unique contributions of executive functions but also the coordinated interplay of these functions. We submit the hypothesis that training the coordinated interplay of executive functions rather than training isolated functions facilitates transfer. The coordinated interplay of executive functions may be best accomplished in real academic or informal learning environments, including complex tasks (e.g., solving statistical problems) that are optimized with respect to their demands on executive functions when necessary. In contrast, isolated computerized trainings with a limited set of stimuli may be less appropriate for addressing the large number of factors potentially involved in improving executive functions (e.g., coordinated interplay of executive functions in several contexts; or social support). In addition, the aim of acquiring relevant domain knowledge is not usually included in these individualized and narrow training programs. Thus, complex activity contexts are promising in that they are intended to help approach the dual goal of advancing domain knowledge and executive functions at the same time. 
We suggest systematically addressing six issues for enhancing executive functions in complex learning activities.

a) Investigating the coordinated interplay of executive functions.

The hypothesis that the coordinated interplay of executive functions is crucial for improving these cognitive functions should be tested empirically. The overarching rationale behind this line of research has to be further elaborated. Isolated trainings of single components of the cognitive system (i.e., training of specific executive functions) with material low in complexity did not show transfer effects to other cognitive abilities (e.g., Schwaighofer et al., 2015; Melby-Lervåg \& Hulme, 2016). One explanation for the missing transfer effects could be that executive functions rarely operate in isolation when learners are faced with complex learning tasks. If complex learning tasks put a consistently high demand on executive functions and especially their coordinated interplay, these functions and their interplay may adapt to high task demands. The adaptation may result in the improvement of single executive functions and importantly the interplay of executive functions. Furthermore, cognitive abilities or skills related to complex learning activities (e.g., reading abilities, learning about physiological, metabolic processes or handling chemical equations) may also improve if they put a similar demand on executive functions operating in parallel. In line with these suggestions, the implementation of training of executive functions within complex activity contexts such as specific learning environments in schools has yielded promising results with respect to the improvement of executive functions and transfer effects to other cognitive abilities (e.g., Blair \& Raver, 2014; Diamond et al., 2007; Raver et al., 2011).

Research on training of executive functions in complex activity contexts can benefit from studies investigating the moderating role of executive functions in learning environments with varying degrees of instructional support and context characteristics. The moderating role of executive functions suggests that the demand on these functions may depend on the degree of instructional support and/or context characteristics. One example is the moderating role of shifting for the effects of worked examples versus problem solving. The moderating role suggests that the demand on shifting is high when solving complex problems, which require frequent shifts among relevant and irrelevant information due to a rather low degree of instructional support (Schwaighofer et al., 2016). Thus, solving complex problems requiring frequent shifts among relevant and irrelevant information could offer a way for improving shifting, especially for learners who are challenged to the limits of their cognitive functions. If shifting, working memory, and inhibition moderate the effects of different learning environments on learning outcomes, their coordinated interplay may be important under particular conditions. Therefore, the moderating role of executive functions in complex activity contexts may inform on the demands of instructional approaches on executive functions and their interplay. Scenarios with a high demand on executive functions and their interplay can serve as fruitful starting points for improving executive functions in the long run.

b) Implementing adaptive instructional support.

The degree of instructional support may be lowered when executive functions improve over time to put a consistently high demand on executive functions. The degree of instructional support can be adaptively reduced depending on the knowledge of learners, such as by fading solution steps of worked examples (e.g., Salden, Aleven, Renkl, \& Schwonke, 2009). However, procedures for fading instructional support depending on the level of executive functions (rather than domain knowledge) have yet to be developed. Learning environments with low versus high demands on executive functions and their interplay can serve as starting points for determining whether putting different demands on executive functions over longer periods of time influences these functions and their coordinated interplay. The effects of these learning environments on executive functions and their interplay should be considered in addition to effects on knowledge acquisition. Further research could compare the effects of instructional approaches adapted to improve executive functions with effects of instructional approaches adapted to foster knowledge acquisition.

c) Testing the cognitive flexibility hypothesis in the context of executive functioning.

The cognitive flexibility hypothesis submits that challenging executive functions in various contexts likely yields transfer effects of improved executive functions to other tasks (Schwaighofer et al., 2015). One way 
of testing this hypothesis would be a comparison of executive function training in various complex contexts with different content materials to training in one context with material similar in content. The various contexts and materials should put different demands on executive functions and their coordinated interplay. The instructional environments would have to establish consistently high demands. This suggestion is in line with the detrimental instructional support hypothesis and the explanations regarding the adaptation of the degree of instructional support detailed above.

d) Testing the superiority of training effects for learners with poor executive functions.

Future studies could also address the principle that learners with poor executive functions gain most from training of these functions. Many instructional support measures designed to support learners with low prior knowledge appear to have helped them more than learners with higher expertise (e.g., Kalyuga, 2013). An open question is whether different levels of prior knowledge and executive functions influence the effects of approaches to improve executive functions in complex activity contexts. These presumably local moderation effects can be tested by applying the Johnson-Neyman technique (Hayes, 2013).

e) Pushing the limits: New forms of assessment of executive functions and their coordinated interplay.

Effects of instructional approaches on executive functions should be measured with tasks that push the limits of participants' executive functions and/or require the interplay of executive functions. In addition to single measures of executive functions that are considered to be independent of domain-specific knowledge (e.g., complex span tasks that measure working memory capacity; see Redick, Broadway, et al., 2012), more knowledge-rich transfer measures should be used. Knowledge-rich transfer measures include problems from specific domains that require the interplay of executive functions and domain knowledge to develop an appropriate solution.

f) Setting the stage through indirect factors influencing executive functions.

Finally, complementary to proximal factors that may influence executive functions in the long run, some rather distal variables seem to have an influence on executive functions and thus should not be neglected. Diamond and Ling (2016) propose that the most successful interventions for improving executive functions will be those that train these functions directly and support them indirectly as well. Indirect support involves minimizing factors that appear to decrease executive functions such as stress or sadness and support factors that appear to enhance executive functions such as joy and social support (Diamond \& Ling, 2016). The long-term impact of factors influencing executive functions such as frustration (Pnevmatikos \& Trikkaliotis, 2013) and motivation (Sosic-Vasic et al., 2015) need to be further addressed in future research.

\section{Conclusion}

To conclude, recent instructional approaches typically focus on the acquisition of academically relevant knowledge with complex learning tasks. Executive functions are important learning prerequisites and need to be more systematically investigated as to their moderating effect on knowledge acquisition under different instructional support conditions. Assuming malleability of executive functions as well, instructional conditions may affect their development, at least in the long run. It is important to know more about the conditions under which instructional approaches influence the development of executive functions. Conditions for optimizing knowledge acquisition (e.g., moderate cognitive load) may be detrimental to the development of executive functions because the demand on these functions is low. It is not yet known whether and how learning environments can be designed that foster domain learning and enhance executive functions at the same time. 


\section{Keypoints}

- Within the frameworks of complex learning and aptitude treatment interaction, we argue that executive functions and other cognitive functions (particularly fluid intelligence) may moderate the effects of various instructional approaches in interaction with prior knowledge.

- We suggest that the moderating role of cognitive prerequisites may depend on the degree of instructional support and context characteristics.

- Based on research on training of executive functions, we propose that complex activity contexts have the potential to improve executive functions and yield practically relevant transfer effects to other cognitive abilities.

6. This article proposes several lines for future research on the interaction of cognitive prerequisites and various instructional approaches as well as the training of executive functions in complex activity contexts.

\section{References}

Ackerman, P. L., Beier, M. E., \& Boyle, M. O. (2002). Individual differences in working memory within a nomological network of cognitive and perceptual speed abilities. Journal of Experimental Psychology. General, 131(4), 567-589. doi: 10.1037/0096-3445.131.4.567

Agostino, A., Johnson, J., \& Pascual-Leone, J. (2010). Executive functions underlying multiplicative reasoning: Problem type matters. Journal of Experimental Child Psychology, 105(4), 286-305. doi: 10.1016/j.jecp.2009.09.006

Andersson, U. (2010). Skill development in different components of arithmetic and basic cognitive functions: Findings from a 3-year longitudinal study of children with different types of learning difficulties. Journal of Educational Psychology, 102(1), 115-134. doi:10.1037/a0016838

Baddeley, A. D., Allen, R. J., \& Hitch, G. J. (2011). Binding in visual working memory: The role of the episodic buffer. Neuropsychologia, 49(6), 1393-1400. doi: 10.1016/j.neuropsychologia.2010.12.042

Berends, I. E., \& van Lieshout, E. C. D. M. (2009). The effect of illustrations in arithmetic problem-solving: Effects of increased cognitive load. Learning and Instruction, 19, 345-353. doi: 10.1016/j.learninstruc.2008.06.012

Blair, C., Knipe, H., \& Gamson, D. (2008). Is there a role for executive functions in the development of mathematics ability? Mind, Brain, and Education, 2(2), 80-89. doi: 10.1111/j.1751-228X.2008.00036.x

Blair, C., \& Raver, C. C. (2014). Closing the achievement gap through modification of neurocognitive and neuroendocrine function: Results from a cluster randomized controlled trial of an innovative approach to the education of children in kindergarten. PLoS ONE, 9(11), e112393. doi: 10.1371/journal.pone.0112393

Blair, C., \& Ursache, A. (2011). A bidirectional theory of executive functions and self-regulation. In K. Vohs, \& R. Baumeister (Eds.), Handbook of self-regulation. (2 ed., pp. 300-320). New York: Guilford Press.

Blair, C., Ursache, A., Greenberg, M., Vernon-Feagans, L., \& The Family Life Project Investigators (2015). Multiple aspects of self-regulation uniquely predict mathematics but not letter-word knowledge in the early elementary grades. Developmental Psychology, 51(4), 459-472. doi: 10.1037/a0038813

Borella, E., \& de Ribaupierre, A. (2014). The role of working memory, inhibition, and processing speed in text comprehension in children. Learning and Individual Differences, 34, 86-92. doi: 10.1016/j.lindif.2014.05.001

Bühner, M., Kröner, S., \& Ziegler, M. (2008). Working memory, visual-spatial-intelligence and their relationship to problem-solving. Intelligence, 36(6), 672-680. doi:10.1016/j.intell.2008.03.008

Bull, R., \& Lee, K. (2014). Executive functioning and mathematics achievement. Child Development Perspectives, 8(1), 36-41. doi: 10.1111/cdep.12059

Cowan, N. (2001). The magical number 4 in short-term memory: A reconsideration of mental storage capacity. 
Behavioral and Brain Sciences, 24(1), 87-185. doi:10.1017/S0140525X01003922

Daneman, M., \& Merikle, P. M. (1996). Working memory and language comprehension: A meta-analysis. Psychonomic Bulletin \& Review, 3(4), 422-433. doi: 10.3758/BF03214546

de Jong, T. (2010). Cognitive load theory, educational research, and instructional design: Some food for thought. Instructional Science, 38, 105-134. doi: 10.1007/s11251-009-9110-0

Diamond, A. (2013). Executive functions. Annual Review of Psychology, 64(1), 135-168. doi: 10.1146/annurevpsych-113011-143750

Diamond, A., Barnett, W. S., Thomas, J., \& Munro, S. (2007). Preschool program improves cognitive control. Science, 318 (5855), 1387-1388. doi: 10.1126/science.1151148

Diamond, A., \& Ling, D. S. (2016). Conclusions about interventions, programs, and approaches for improving executive functions that appear justified and those that, despite much hype, do not. Developmental Cognitive Neuroscience, 18, 34-48. doi: 10.1016/j.den.2015.11.005

Dochy, F., Segers, M., \& Buehl, M. M. (1999). The relation between assessment practices and outcomes of studies: The case of research on prior knowledge. Review of Educational Research, 69(2), 145-186. doi:10.3102/00346543069002145

Draheim, C., Hicks, K. L., \& Engle, R. W. (2016). Combining reaction time and accuracy: The relationship between working memory capacity and task switching as a case example. Perspectives on Psychological Science, 11(1), 133-155. doi: 10.1177/1745691615596990

Ecker, U. K. H., Lewandowsky, S., \& Oberauer, K. (2014). Removal of information from working memory: A specific updating process. Journal of Memory and Language, 74, 77-90. doi: 10.1016/j.jml.2013.09.003

Ecker, U. K. H., Lewandowsky, S., Oberauer, K., \& Chee, A. E. H. (2010). The components of working memory updating: An experimental decomposition and individual differences. Journal of Experimental Psychology: Learning, Memory, and Cognition, 36(1), 170-189. doi: 10.1037/a0017891

Espy, K. A., McDiarmid, M. M., Cwik, M. F., Stalets, M. M., Hamby, A., \& Senn, T. E. (2004). The contribution of executive functions to emergent mathematic skills in preschool children. Developmental Neuropsychology, 26, 465-486. doi: 10.1207/s15326942dn2601_6

Eysenck, M. W., Derakshan, N., Santos, R., \& Calvo, M. G. (2007). Anxiety and cognitive performance: Attentional control theory. Emotion, 7(2), 336-353. doi: 10.1037/1528-3542.7.2.336

Friedman, N. P., \& Miyake, A. (2004). The relations among inhibition and interference control functions: A latent-variable analysis. Journal of Experimental Psychology. General, 133(1), 101-135. doi:10.1037/00963445.133.1.101

Friedman, N. P., \& Miyake, A. (2017). Unity and diversity of executive functions: Individual differences as a window on cognitive structure. Cortex, 86, 186-204. doi: 10.1016/j.cortex.2016.04.023

Friedman, N. P., Miyake, A., Altamirano, L. J., Corley, R. P., Young, S. E., Rhea, S. A., \& Hewitt, J. K. (2016). Stability and change in executive function abilities from late adolescence to early adulthood: A longitudinal twin study. Developmental Psychology, 52(2), 326-340. doi: 10.1037/dev0000075

Friedman, N. P., Miyake, A., Robinson, J. L., \& Hewitt, J. K. (2011). Developmental trajectories in toddlers' self-restraint predict individual differences in executive functions 14 years later: A behavioral genetic analysis. Developmental Psychology, 47(5), 1410-1430. doi: 10.1037/a0023750

Friedman, N. P., Miyake, A., Young, S. E., DeFries, J. C., Corley, R. P., \& Hewitt, J. K. (2008). Individual differences in executive functions are almost entirely genetic in origin. Journal of Experimental Psychology: General, 137(2), 201-225. doi: 10.1037/0096-3445.137.2.201

Gathercole, S. E., \& Alloway, T. P. (2007). Understanding working memory. A classroom guide. London, UK: Harcourt Assessment. Retrieved from http://www.york.ac.uk/res/wml/Classroom\%20guide.pdf

Hayes, A. F. (2013). Introduction to mediation, moderation, and conditional process analysis: A regressionbased approach. New York: Guilford Press.

Holmes, J., Gathercole, S. E., Place, M., Dunning, D. L., Hilton, K. A., \& Elliott, J. G. (2010). Working memory deficits can be overcome: Impacts of training and medication on working memory in children with ADHD. Applied Cognitive Psychology, 24(6), 827-836. doi: 10.1002/acp.1589

Jacob, R., \& Parkinson, J. (2015). The potential for school-based interventions that target executive function to improve academic achievement: A review. Review of Educational Research, 85(4), 512-552. doi: 


\section{$10.3102 / 0034654314561338$}

Jacobson, L. A., Koriakin, T., Lipkin, P., Boada, R., Frijters, J. C., Lovett, M. W., ... Mahone, E. M. (2016). Executive functions contribute uniquely to reading competence in minority youth. Journal of Learning Disabilities. doi: 10.1177/0022219415618501

Jaeggi, S. M., Buschkuehl, M., Jonides, J., \& Perrig, W. J. (2008). Improving fluid intelligence with training on working memory. Proceedings of the National Academy of Sciences, 105(19), 6829-6833.

Kalyuga, S. (2007). Expertise reversal effect and its implications for learner-tailored instruction. Educational Psychology Review, 19, 509-539. doi: 10.1007/s10648-007-9054-3

Kalyuga, S. (2013). Effects of learner prior knowledge and working memory limitations on multimedia learning. Procedia - Social and Behavioral Sciences, 83, 25-29. doi:10.1016/j.sbspro.2013.06.005

Kalyuga, S., Rikers, R., \& Paas, F. (2012). Educational implications of expertise reversal effects in learning and performance of complex cognitive and sensorimotor skills. Educational Psychology Review, 24(2), 313337. doi: 10.1007/s10648-012-9195-x

Kalyuga, S., \& Singh, A.-M. (2015). Rethinking the boundaries of cognitive load theory in complex learning. Educational Psychology Review. doi: 10.1007/s10648-015-9352-0

Kalyuga, S., \& Sweller, J. (2004). Measuring knowledge to optimize cognitive load factors during instruction. Journal of Educational Psychology, 96(3), 558-568. doi: 10.1037/0022-0663.96.3.558

Karbach, J., \& Verhaeghen, P. (2014). Making working memory work: A meta-analysis of executive-control and working memory training in older adults. Psychological Science, 25(11), 2027-2037. doi: $10.1177 / 0956797614548725$

Kester, L., \& Kirschner, P. A. (2012). Cognitive tasks and learning. In N. Seel (Ed.), Encyclopedia of the Sciences of Learning (pp. 619-622). New York: Springer.

König, C. J., Bühner, M., \& Mürling, G. (2005). Working memory, fluid intelligence, and attention are predictors of multitasking performance, but polychronicity and extraversion are not. Human Performance, 18(3), 243-266. doi:10.1207/s15327043hup1803_3

Kramer, A. F., \& Erickson, K. I. (2007). Capitalizing on cortical plasticity: Influence of physical activity on cognition and brain function. Trends in Cognitive Sciences, 11(8), 342-348. doi: 10.1016/j.tics.2007.06.009

Krumm, S., Schmidt-Atzert, L., Bühner, M., Ziegler, M., Michalczyk, K., \& Arrow, K. (2009). Storage and nonstorage components of working memory predicting reasoning: A simultaneous examination of a wide range of ability factors. Intelligence, 37(4), 347-364. doi:10.1016/j.intell.2009.02.003

Lan, X., Legare, C., Ponitz, C. C., Li, S., \& Morrison, F. J. (2011). Investigating the links between the subcomponents of executive function and academic achievement: A cross-cultural analysis of Chinese and American preschoolers. Journal of Experimental Child Psychology, 108, 677-692. doi:10.1016/j.jecp.2010.11.001

Lee, K., Bull, R., \& Ho, R. M. H. (2013). Developmental changes in executive functioning. Child Development, 84, 1933-1953. doi: 10.1111/cdev.12096

Lehmann, J., Goussios, C., \& Seufert, T. (2016). Working memory capacity and disfluency effect: An aptitudetreatment-interaction study. Metacognition and Learning, 11(1), 89-105. doi: 10.1007/s11409-015-9149-z

Liston, C., McEwen, B. S., \& Casey, B. J. (2009). Psychosocial stress reversibly disrupts prefrontal processing and attentional control. Proceedings of the National Academy of Sciences, 106(3), 912-917. doi: 10.1073/pnas.0807041106

Melby-Lervåg, M., \& Hulme, C. (2016). There is no convincing evidence that working memory training is effective: A reply to Au et al. (2014) and Karbach and Verhaeghen (2014). Psychonomic Bulletin \& Review, 23(1), 324-330. doi: 10.3758/s13423-015-0862-z

Melby-Lervåg, M., Redick, T. S., \& Hulme, C. (2016). Working memory training does not improve performance on measures of intelligence or other measures of "far transfer": Evidence from a meta-analytic review. Perspectives on Psychological Science, 11 (4), 512-534. doi: 10.1177/1745691616635612

Mischel, W., Ayduk, O., Berman, M. G., Casey, B. J., Gotlib, I. H., Jonides, J., ... Shoda, Y. (2011). "Willpower" over the life span: Decomposing self-regulation. Social Cognitive and Affective Neuroscience, 6(2), 252-256. doi:10.1093/scan/nsq081

Miyake, A., \& Friedman, N. P. (2012). The nature and organization of individual differences in executive 
functions: Four general conclusions. Current Directions in Psychological Science, 21(1), 8-14. doi:10.1177/0963721411429458

Miyake, A., Friedman, N. P., Emerson, M. J., Witzki, A. H., Howerter, A., \& Wager, T. D. (2000). The unity and diversity of executive functions and their contributions to complex "frontal lobe" tasks: A latent variable analysis. Cognitive Psychology, 41, 49-100. doi: 10.1006/ cogp.1999.0734

Oberauer, K., Schulze, R., Wilhelm, O., \& Süß, H. M. (2005). Working memory and intelligence-Their correlation and their relation: Comment on Ackerman, Beier, and Boyle (2005). Psychological Bulletin, 131, 61-65. doi: 10.1037/0033-2909.131

Oswald, F. L., McAbee, S. T., Redick, T. S., \& Hambrick, D. Z. (2015). The development of a short domaingeneral measure of working memory capacity. Behavior Research Methods, 47(4), 1343-1355. doi: 10.3758/s13428-014-0543-2

Park, B., Korbach, A., \& Brünken, R. (2015). Do learner characteristics moderate the seductive-details-effect? A cognitive-load-study using eye-tracking. Journal of Educational Technology \& Society, 18(4), 24-36.

Peng, P., Namkung, J., Barnes, M., \& Sun, C. (2016). A meta-analysis of mathematics and working memory: Moderating effects of working memory domain, type of mathematics skill, and sample characteristics. Journal of Educational Psychology, 108(4), 455-473. doi: 10.1037/edu0000079

Pnevmatikos, D., \& Trikkaliotis, I. (2013). Intraindividual differences in executive functions during childhood: The role of emotions. Journal of Experimental Child Psychology, 115(2), 245-261. doi: 10.1016/j.jecp.2013.01.010

Primi, R., Ferrão, M. E., \& Almeida, L. S. (2010). Fluid intelligence as a predictor of learning: A longitudinal multilevel approach applied to math. Learning and Individual Differences, 20(5), 446-451. doi:10.1016/j.lindif.2010.05.001

Redick, T. S., Broadway, J. M., Meier, M. E., Kuriakose, P. S., Unsworth, N., Kane, M. J., \& Engle, R. W. (2012). Measuring working memory capacity with automated complex span tasks. European Journal of Psychological Assessment, 28, 164-171. doi: 10.1027/ 1015 5759/a000123

Redick, T. S., Unsworth, N., Kelly, A. J., \& Engle, R. W. (2012). Faster, smarter? Working memory capacity and perceptual speed in relation to fluid intelligence. Journal of Cognitive Psychology, 24, 844-854. doi: $10.1080 / 20445911.2012 .704359$

Renkl, A. (2014). Toward an instructionally oriented theory of example-based learning. Cognitive Science, 38(1), 1-37. doi:10.1111/cogs.12086

Salden, R. J., Aleven, V. A., Renkl, A., \& Schwonke, R. (2009). Worked examples and tutored problem solving: Redundant or synergistic forms of support? Topics in Cognitive Science, 1(1), 203-213.

Schmiedek, F., Hildebrandt, A., Lövdén, M., Wilhelm, O., \& Lindenberger, U. (2009). Complex span versus updating tasks of working memory: The gap is not that deep. Journal of Experimental Psychology: Learning, Memory, and Cognition, 35(4), 1089-1096. doi: 10.1037/a0015730

Schmiedek, F., Lövdén, M., \& Lindenberger, U. (2014). A task is a task is a task: putting complex span, n-back, and other working memory indicators in psychometric context. Frontiers in Psychology, 5. doi: $10.3389 /$ fpsyg.2014.01475

Schnotz, W., \& Kürschner, C. (2007). A reconsideration of cognitive load theory. Educational Psychology Review, 19(4), 469-508. doi: 10.1007/s10648-007-9053-4

Schwaighofer, M., Bühner, M., \& Fischer, F. (2016). Executive functions as moderators of the worked example effect: When shifting is more important than working memory capacity. Journal of Educational Psychology, 108(7), 982-1000. https://doi.org/10.1037/edu0000115

Schwaighofer, M., Fischer, F., \& Bühner, M. (2015). Does working memory training transfer? A meta-analysis including training conditions as moderators. Educational Psychologist, 50(2), 138-166. doi: $10.1080 / 00461520.2015 .1036274$

Seufert, T., Schütze, M., \& Brünken, R. (2009). Memory characteristics and modality in multimedia learning: An aptitude-treatment-interaction study. Learning and Instruction, 19(1), 28-42. http://doi: 10.1016/j.learninstruc.2008.01.002

Shipstead, Z., Lindsey, D. R. B., Marshall, R. L., \& Engle, R. W. (2014). The mechanisms of working memory capacity: Primary memory, secondary memory, and attention control. Journal of Memory and Language, 
72, 116-141. doi: 10.1016/j.jml.2014.01.004

Snow, R. E., \& Lohman, D. (1984). Toward a theory of cognitive aptitude for learning from instruction. Journal of Educational Psychology, 76, 347-376.

Sosic-Vasic, Z., Keis, O., Lau, M., Spitzer, M., \& Streb, J. (2015). The impact of motivation and teachers'autonomy support on children's executive functions. Frontiers in Psychology, 6. doi: 10.3389/fpsyg.2015.00146

Spiro, R. J., Coulson, R. L., Feltovich, P. J., \& Anderson, D. K. (1988). Cognitive flexibility theory: Advanced knowledge acquisition in illstructured domains. In Proceedings of the Tenth Annual Conference of the Cognitive Science Society (pp. 375-383). Hillsdale, NJ: Erlbaum.

Sweller, J. (2010). Element interactivity and intrinsic, extraneous, and germane cognitive load. Educational Psychology Review, 22(2), 123-138. doi: 10.1007/s10648-010-9128-5

Sweller, J. (2011). Cognitive load theory. In J. Mestre, \& B. Ross (Eds.), The psychology of learning and motivation: Cognition in education (Vol. 55, pp. 37 - 76). Oxford: Academic Press.

Sweller, J., Mawer, R. F., \& Howe, W. (1982). Consequences of history-cued and means-end strategies in problem solving. American Journal of Psychology, 95, 455-483. doi: 10.2307/1422136

Trezise, K., \& Reeve, R. A. (2014). Cognition-emotion interactions: patterns of change and implications for math problem solving. Frontiers in Psychology, 5. doi: 10.3389/fpsyg.2014.00840

Tsaparlis, G. (2005). Non-algorithmic quantitative problem solving in university physical chemistry: A correlation study of the role of selective cognitive factors. Research in Science \& Technological Education, 23, 125-148. doi: 10.1080/02635140500266369

Tun, P. A., Miller-Martinez, D., Lachman, M. E., \& Seeman, T. (2013). Social strain and executive function across the lifespan: The dark (and light) sides of social engagement. Aging, Neuropsychology, and Cognition, 20(3), 320-338. doi: 10.1080/13825585.2012.707173

van Gog, T., \& Rummel, N. (2010). Example-based learning: Integrating cognitive and social-cognitive research perspectives. Educational Psychology Review, 22(2), 155-174. doi:10.1007/s10648-010-9134-7

van Merriënboer, J. J. G., \& Kirschner, P. A. (2012). Ten steps to complex learning: A systematic approach to four-component instructional design (2nd ed.). New York: Routledge.

Wilhelm, O., Hildebrandt, A., \& Oberauer, K. (2013). What is working memory capacity, and how can we measure it? Frontiers in Psychology, 4. doi: 10.3389/fpsyg.2013.00433

Yeniad, N., Malda, M., Mesman, J., van Ijzendoorn, M. H., \& Pieper, S. (2013). Shifting ability predicts math and reading performance in children: A meta-analytical study. Learning and Individual Differences, 23, 19. doi:10.1016/j.lindif.2012.10.004 